\title{
Evaluation of High-Resolution Melting Curve Analysis (HRM) assay for Detection of Pseudomonas aeruginosa PASGNDM699: A dangerous New Delhi metallo- $\beta$-lactamase (NDM) strain
}

\section{Sanaz Dehbashi}

Hamadan University of Medical Sciences Medical School

Hamed Tahmasebi

Hamadan University of Medical Sciences Medical School

Mohammad Arabestani ( $\nabla$ mohammad.arabestani@gmail.com )

Hamadan University of Medical Sciences Medical School

\section{Research article}

Keywords: Pseudomonas aeruginosa, High-Resolution Melting Curve Analysis (HRM), $\beta$-lactamases,

Resistance rate

Posted Date: November 7th, 2019

DOl: https://doi.org/10.21203/rs.2.16996/v1

License: (c) (1) This work is licensed under a Creative Commons Attribution 4.0 International License. Read Full License 


\section{Abstract}

Background: New Delhi metallo- $\beta$-lactamase (NDM-1) is a broad spectrum $\beta$-lactamase that is able to inactivate all $\beta$-lactams except aztreonam, as is typical of metallo- $\beta$-lactamases. NDM-1 producers in Pseudomonas aeruginosa, especially PASGNDM699 strain, cause a range of infections such as urinary tract, diarrhoea and soft tissue infections. The aim of this study was to Standardization of HighResolution Melting Curve Analysis (HRM) assay for detection of P. aeruginosa, especially PASGNDM699 strain. Methods: The HRM method was done on standard strains of P. aeruginosa strains. 9-fold Serial dilutions of known DNA concentrations, extracted from standard isolates were prepared and tested by Real Time Melting curve and HRM assay. Data analysis was performed using the StepOne Software v2.3 and HRM Software v3.0.1 (Applied Biosystems, Ltd). Results: Based on the results of the Real Time PCR assay and melt curve analysis, melting point temperatures of the $\mathrm{N}-1, \mathrm{~N}-2$ and $\mathrm{N}-3$ amplicon for isolates identified as NDM strains were $87.57^{\circ} \mathrm{C}, 76.92^{\circ} \mathrm{C}$ and $82.97^{\circ} \mathrm{C}$, respectively. Furthermore, melting point temperatures of the blaVIM, blaSPM and blaSIM amplicon for isolates identified as MBL strains were $84.56^{\circ} \mathrm{C}, 85.35^{\circ} \mathrm{C}$ and $86.62^{\circ} \mathrm{C}$, respectively. Due to the analytical specificity of the primers, all dilutions with a similar Tm and melt peaks were obtained in the melting curves. Moreover, the analytical sensitivity of NDM primer were able to detected $100 \mathrm{CFU} / \mathrm{mL}, 103 \mathrm{CFU} / \mathrm{mL}$ and $104 \mathrm{CFU} / \mathrm{mL}$ of standard DAN by $\mathrm{N}-1$, $\mathrm{N}-2$ and N-3 primers, respectively. Also, according to analytical sensitivity of MBL primers, blaVIM was able detected of $100 \mathrm{CFU} / \mathrm{mL}$, blaSPM primer $105 \mathrm{CFU} / \mathrm{mL}$ and blaSIM primer $102 \mathrm{CFU} / \mathrm{mL}$ of PASGNDM699 strain. HRM results showed that N-1 primers with $55 \mathrm{bp}$ and blaVIM primers with $124 \mathrm{bp}$ had the highest sensitivity and specificity for P. aeruginosa PASGNDM699 strain identification. Conclusion: The data from our study indicated that the sensitivity and specificity of the HRM method linked to the primer length and the fluorescent dye. Further, we can identify antibiotic resistance in substrates such as P. aeruginosa PASGNDM699 by software analysis and melting curve analysis.

\section{Background}

Pseudomonas aeruginosa is one of the major microorganisms involved in urinary, bloodstream, pulmonary, soft tissue, and surgical site infections in compromised individuals as those in intensive care units[1]. $\beta$-lactam antibiotics make up more than $50 \%$ of all commercially prescribed antibiotics for treatment of bacterial infections. The general mechanism of action of $\beta$-lactam antibiotics is inhibition of peptidoglycan synthesis which constitutes a major portion of bacterial cell wall synthesis[2]. The attachment of $\beta$-lactam antibiotics to penicillin-binding protein leads to the inhibition of transpeptidase that eventually leads to the death of bacteria. Several mechanisms to attain resistance against $\beta$-lactam antibiotics $[3,4]$. These include: mutations to the active site of penicillin-binding-protein(PBP) to prevent drug binding, modification of the cell wall to prevent drug entry and assist active removal of antibiotic compounds, and producing the class of enzyme known as $\beta$-lactamase, which includes serine $\beta$ lactamases and metallo- $\beta$-lactamase (MBLs) $[5,6]$. These strains hydrolyze the $\beta$-lactam ring of drug compound, thereby inactivating them. In contrast to serine $\beta$-lactamases, MBLs use at least one but more commonly two $\mathrm{Zn} 2+$ ions in their active site to catalyze the hydrolysis of $\beta$-lactam rings $[2,3]$. There are 
various methods for identifying MBL and NDM strains, which fall into two phenotypic and genotypic groups. Usually, phenotypic methods have low specificity, low speed, and error in results. Therefore, it is necessary to use molecular methods along with phenotypic methods. High-resolution melt (HRM) analysis is one of the most sensitive and precise molecular methods based on Real Time PCR[7].

HRM is used to characterize samples according to their dissociation behavior as they transition from dsDNA to ssDNA with increasing temperature and fluorescence detection. HRM melt curves is a function of the amplicon DNA sequence, which allows discrimination of amplicons with different nucleotide sequence based on melt curve shape, regardless of the amplicon $\operatorname{Tm}[8,9]$. The melt curve analysis is generally used in conjunction with HRM. It is generated after PCR amplification and indicates a change in fluorescence as temperature is raised by a fraction of a degree, from $60^{\circ} \mathrm{C}$ and slowly increasing to $95^{\circ} \mathrm{C}[10,11]$. Quenched amplicons produce a significant change in fluorescent signal, as they are denatured. The difference in fluorescence is used to determine the melting temperature (Tm), the temperature at which amplicon dissociation occurs. HRM and that the melting temperature $(\mathrm{Tm})$ of PCR products can vary based on the length and DNA sequence[12,13]. This method has been shown to provide a successful platform for the identification of microbial pathogens, and for discriminating Single Nucleotide Polymorphisms (SNPs) that confer a decrease in susceptibility to antimicrobials. The bases " $G$ " and " $C$ " have 3 hydrogen bonds connecting them whereas the bases " $A$ " and " $T$ " have only $2[10,13]$. This small difference will affect the melting temperature in which the bond will break by requiring a higher melting temperature for DNA with larger amounts of GC content[14]. HRM has the potential to be a powerful tool in the clinical microbiology laboratory, providing rapid detection of genetic determinants conferring antibiotic resistance to complement current phenotypic antimicrobial susceptibility testing methods[15].

The overarching aim of the current study was to define the genetic events that take place when resistance to third generation $\beta$-lactamase is selected in PASGNDM699 strain of $P$. aeruginosa by HRM technique.

\section{Methods}

\section{Study design and $P$. aeruginosa strains}

Standard cultures of $P$. aeruginosa PASGNDM699 (detection in clinical isolates), Enterococcus faecalis NCTC13779 and Staphylococcus aureus ATCC25923 were purchased from Pasteur Institute (Tehran, Iran). Cultures were grown at $37^{\circ} \mathrm{C}$ in Tryptic Soy broth (Merck, Germany). Biochemical tests were used to differentiate between closely related bacterial species or genus. These tests were conducted according to the standard specifications described and applied in the Microbiology Laboratory at Hamedan medical science. P. aeruginosa colonies were identified based on morphology, Gram staining, pyocyanin production and a biochemical test (such as oxidase, catalase, urease, arginine dehydrogenase and oxidation-fermentation). Bacterial strains were stored at $-20^{\circ} \mathrm{C}$ in Brain-Heart Infusion (BHI) broth supplemented with $25 \% \mathrm{v} / \mathrm{v}$ glycerol. This study was approved by the Ethics Committee of Hamadan University of Medical Sciences (Code No: IR.UMSHA.REC.1396.637). 


\section{DNA Extraction, PCR assay and Sequencing}

P. aeruginosa DNA extraction was performed using the DNA extraction kit (Qiagen, Germany), the steps were followed according to the kit protocol. DNA concentration was determined using a spectrophotometer Nanodrop-200 (Hangzhou Allsheng Instruments Co., Ltd, China). Primers sequence were initially set up as outlined in Ly et al. [16], Bordin et al. [6], Monteiro et al. [17], Kosykowska et al[18], and Alkasaby et al[19]. PCR amplification was done in $50 \mu$ reaction volumes containing $0.5 \mu \mathrm{M}$ of each primer, $25 \mu \mathrm{M} 2 \mathrm{X}$ mastermixe (Ampliqon, Denmark) $1 \mu \mathrm{l}$ DNA extract, and $5 \mu \mathrm{l}$ of supplied deionized water. Amplification was done using PCR thermocycler 1001C (BioRad, Germany) using the following profile: initial denaturation at $95^{\circ} \mathrm{C}$ for $5 \mathrm{~min}, 30$ cycles of denaturation at $95^{\circ} \mathrm{C}$ for $1 \mathrm{~min}$, annealing at $61^{\circ} \mathrm{C}$ for $1 \mathrm{~min}$, and extension at $72^{\circ} \mathrm{C}$ for $1 \mathrm{~min}$, and a final extension at $72^{\circ} \mathrm{C}$ for $5 \mathrm{~min}$. Amplified products were detected by agarose gel electrophoresis in 1\% Tris-Acetate-EDTA (TAE) agarose (Sigma-Aldrich, USA). In this study, we performed the Sangar chain termination method for sequencing for all genes. All PCR products were sent to Pishgam Company (Tehran, Iran) for sequencing.

\section{Real-time PCR and primers sensitivity and specificity}

Primers sequence were initially set up as outlined in Sekyere (Osei Sekyere 2019) and Lee and Ko (Lee and Ko; 2014). For sensitivity and specificity of primers, nine-fold serial dilutions of $0.5 \mathrm{McFarland}$ DNA $\left(1.5 \times 10^{8} \mathrm{CFU} / \mathrm{mL}\right)$ were made $\left(1: 1^{-1}, 1: 1^{-2}, 1: 1^{-3}, 1: 1^{-4}, 1: 1^{-5}, 1: 1^{-6}, 1: 1^{-7}, 1: 1^{-8}\right)$. Primers efficiencies of both target genes and reference genes were tested by serial dilution Real-time PCR. Standard curves were constructed by the $\mathrm{Ct}$ (y-axis) versus log DNA dilution ( $\mathrm{x}$-axis). The primer efficiency (E) of one cycle in the exponential phase was calculated according to the equation: $E=10-(1 /$ slope) $-1 \times 100[20]$. Briefly, $2 \mu \mathrm{L} 0.5 \mu \mathrm{M}$ of each primer, $2 \mu \mathrm{L}$ DNA template, $4 \mu \mathrm{L}$ EvaGreen, and made up to a final volume of $20 \mu \mathrm{L}$ using ddH2O. Real-time PCR reactions were performed on the ABI Real-time machine (ABI step one plus, USA). The thermal cycles were set for reverse transcription steps: $55^{\circ} \mathrm{C}$ for $5 \mathrm{~min}, 95^{\circ} \mathrm{C}$ for $10 \mathrm{~min}, 95^{\circ} \mathrm{C}$ for $20 \mathrm{sec}$ followed by PCR steps: $95^{\circ} \mathrm{C}$ for $15 \mathrm{sec}, 59^{\circ} \mathrm{C}$ for $30 \mathrm{sec}$ repeated for 40 cycles. An arbitrary threshold value of 0.002 was set for all real-time PCR reactions. This value conferred a threshold that crossed the real-time PCR amplification curves during the exponential phase of amplification, enabling the determination of a cycle time (CT) value for each reaction. Strains included Enterococcus faecalis NCTC13779 and Staphylococcus aureus ATCC25923 were utilized as controls to assess analytical specificity. To calculate the efficiency of reaction, the slope value was calculated from serial dilutions for each gene, which was then used to determine the efficiency of reaction. In order to obtain accurate and reproducible results, reactions should have efficiency close to $2(100 \%)$, which means that the template doubles with each cycle during exponential amplification. A slope of - 3.32 indicates optimal PCR efficiency.

\section{Evaluation of sensitivity and specificity of HRM assay}

The efficiency and the analytical sensitivity of the HRM-PCR were evaluated by triplicate testing of a 10fold serial dilution series of each of the three reference strains. The standard curve was constructed by 
using the serially diluted DNA of $0.5 \mathrm{McF}$ arland $\left(1 / 5 \times 10^{8} \mathrm{CFU} / \mathrm{mL}\right)$ preparation as template in the optimized HRM assay. A negative control was included in very run and HRM curve assay was performed after amplification to confirm the Tm of the amplification product. The Applied Biosystems step one plus, Real-Time PCR System was used to amplify and detect products. The reaction mix was prepared using the following components for each of the samples: $4 \mu$ l of Master Mix HRM (HOT FIREPol EvaGreen HRM Mix), $1 \mu \mathrm{M}$ of each respective primer and $12 \mu \mathrm{L}$ of DMSO (Sigma-Aldrich, USA). Each reaction contained 2 $\mu \mathrm{l}$ of DNA diluted 1:10 in nuclease-free water. The following cycle parameters were used: $2 \mathrm{~min}$ at $50^{\circ} \mathrm{C}$, $10 \mathrm{~min}$ at $95^{\circ} \mathrm{C}$, followed by 40 cycles with denaturing for $15 \mathrm{sec}$ at $95^{\circ} \mathrm{C}$ and by annealing/elongation for $1 \mathrm{~min}$ at $60^{\circ} \mathrm{C}$. Melting curves were generated after each run to confirm a single PCR product (from $60^{\circ} \mathrm{C}$ to $95^{\circ} \mathrm{C}$, increasing $1^{\circ} \mathrm{C} / 3 \mathrm{sec}$ ).

\section{Data analysis}

Sequencing results were analyzed by BioEdit 7.4 software (Caredata, Inc, USA) to identify the sequences that are reliable and conclusive for mutational analysis and to eliminate sequences with noises that give inconclusive and unreliable data. Peak Tm Calling Analysis was performed after each real-time PCR reaction run within the ABI Thermo Fisher software (release 2018, Version 3.0.2.), based on which clear melting peaks and shoulders were revealed for the amplicons. The temperatures at the melting peaks are recorded as Tm values. Reliable and conclusive sequences obtained after analysis were used to locate the mutation using nucleotide BLAST tool of the National Center for Biotechnology Information (NCBI). Reagent blanks were used to ensure there was no contamination in the reagents.

\section{Results}

\section{Species Identification by PCR and Sequencing:}

Sequencing of standard strains was determined for P. aeruginosa PASGNDM699 with CP020704 accession number.

\section{Analytical Sensitivity and Specificity of Primers:}

Due to the use of 9-fold dilutions, a high CT was observed in the $10^{\circ} \mathrm{CFU} / \mathrm{mL}$ and low CT in the $10^{8}$ $\mathrm{CFU} / \mathrm{mL}$. The CT values for these cell densities were within the 9 to 40 cycle range in the amplification process, while higher DNA concentrations appeared within 9 to 31 cycles. As seen in Figure 1 and Figure 2 , relative to linear range of each standard curve, melting peaks can be seen for many of the lower DNA concentrations, however the concentrations could not be reliably quantified. The actual quantitative, linear portion of the calibration curves therefore did not extend as low as the limit of detection. Melting curves displayed a single melting $\mathrm{Tm}, 87.57^{\circ} \mathrm{C}$ for $\mathrm{N}-1$ gene, $76.92^{\circ} \mathrm{C}$ for $\mathrm{N}-2$ gene, $82.97^{\circ} \mathrm{C}$ for $\mathrm{N}-3$ gene, $84.56^{\circ} \mathrm{C}$ for b/aVIM gene, $86.62^{\circ} \mathrm{C}$ for blaSIM gene and $85.35^{\circ} \mathrm{C}$ for blaSPM gene (Figs 1 and 2). Samples containing DNA exhibit positive real time PCR amplification and negative controls failed to show amplification, also, Serial dilutions of positive control DNA amplification curves showed Ct values that were inversely related to the concentration of template DNA (Fig 3). 
Reaction efficiencies were found to be within the range of 3 to 3.5 when calculated from the standard curves using the $A B I$ Thermo Fisher analysis software (Version 2.3.2) with a formula of $E=10^{(-1 / \mathrm{slope})}-1$. For the N-1, N-2 and blaVIM primer set, the reaction efficiency reached a value slightly greater than 3 , at 3.2, which would suggest an efficiency of $101 \%$. Efficiencies greater than $100 \%$ can be obtained. All the investigated dilutions showed low efficiencies: $\mathrm{N}-3, \mathrm{E}=98.8 \%$; blaSMP, $\mathrm{E}=95.588 \%$ and blaSIM, $E=96.493$ (Figs 1 and 2).

For the $\mathrm{N}-1$ and blaVIM primer set, the linear range was determined to extend as low as $10^{\circ} \mathrm{CFU} / \mathrm{mL}, \mathrm{N}-2$ was $10^{3} \mathrm{CFU} / \mathrm{mL}, \mathrm{N}-3$ was $10^{4} \mathrm{CFU} / \mathrm{mL}$, blaSMP was $10^{2} \mathrm{CFU} / \mathrm{mL}$ and b/aSIM was $10^{5} \mathrm{CFU} / \mathrm{mL}$ as indicated by the lowest DNA concentration value on each of the standard curves. Points which caused the curves to deviate from linearity (mostly those with lower concentrations) were excluded (Figs 1 and2).

\section{Sensitivity and specificity of HRM Assay:}

Fluorescence data were analysed using the tools for HRM analysis incorporated in the ABI Thermo Fisher analysis software. HRM PCR amplification curves of samples analyzed for the presence of $P$. aeruginosa PASGNDM699 are shown in Figure 4, 5 and 6. Difference plots of normalized data show the difference in fluorescence between each sample of DNA. Derivative plots display the rate of fluorescence change; the peak indicates the melting temperature of a sample. All plot displayed a single melting domain, typically between $87.07^{\circ} \mathrm{C}-87.57^{\circ} \mathrm{C}$ for $\mathrm{N}-1$ gene, $76.42^{\circ} \mathrm{C}-76.92^{\circ} \mathrm{C}$ for $\mathrm{N}-2$ gene, $82.47^{\circ} \mathrm{C}-82.97^{\circ} \mathrm{C}$ for $\mathrm{N}-3$ gene, $84.06^{\circ} \mathrm{C}-84.56^{\circ} \mathrm{C}$ for blaVIM gene, $86.12^{\circ} \mathrm{C}-86.62^{\circ} \mathrm{C}$ for blaSIM gene and $85.30^{\circ} \mathrm{C}-85.35^{\circ} \mathrm{C}$ for blaSPM gene in accordance with different product sizes.

The results of this representative experiment show that all samples containing $P$. aeruginosa DNA had measurable amplification as detected by exponential fluorescence and all samples containing negative control DNA did not (Fig 3) and all the DNA dilutions of $P$. aeruginosa PASGNDM699 were identified (dilution $10^{8}$ to $10^{0} \mathrm{CFU} / \mathrm{mL}$ ). In addition, the $\mathrm{N}-1$ and blaVIM genes in $P$. aeruginosa PASGNDM699 was detected in all dilution of DNA. Moreover, N-2, N-3, blaSPM and blaSIM primers can able to detect bacterial DNA in dilutions of $10^{3} \mathrm{CFU} / \mathrm{mL}, 10^{4} \mathrm{CFU} / \mathrm{mL}, 10^{2} \mathrm{CFU} / \mathrm{mL}$, and $10^{5} \mathrm{CFU} / \mathrm{mL}$, respectively (Figs 4 and 5).

The software automatically analyzed the raw melting curve data and set the starting (pre-melt) and ending (post-melt) fluorescence signals of all data to uniform values to aid interpretation and analysis (Figs 4 and 5). The cursors for these two points are defaulted to the ends of the curve but these regions were manually adjusted to encompass representative baseline for the pre-melt and post-melt phases. Widening the normalization regions into the melt phase was avoided to ensure that curves normalize effectively. Moreover, we performed a melt curve analysis of HRM PCR samples to assess the specificity of the amplicon. The results of the HRM showed a very similar melt peak for all Serial dilutions of $P$. aeruginosa.

\section{Discussion}


present study revealed that the sensitivity and specificity of the method for identifying resistant strains have a significant impact on the speed and accuracy of detection. However, to obtain specificity of the Real Time PCR and primers, DNA melting curve analysis (MCA) and standard curve in different dilutions were used. The efficiency of the dilution for all genes was at least $99.99 \%$, the $r 2$ was $>0.99 .99$, and melt curves yielded single peaks. These features are exemplified in Figure 1 and 2 showing the difference between a nonoptimized and optimized standard curve. Interestingly, the slope of the Ct vs DNA relationship varied little across the 9 fold- dilution tested, ranging from -3.589 to -3.955 . Also, No amplification, i.e. CT > 40, was obtained for P. aeruginosa PASGNDM699 strain. According to Lalonde et al[21] and Heydari el al[22] studies, this can be justified because short fragment binds less fluorescent and compensated by its higher primer concentration. However, sometimes the peak height of short amplicon increases in different replicate. This problem gets extremely worse in the MCA that sometimes we lost the long amplicon even at the primer ratio of 1:1. Furthermore, this results agree with Mentasti et al[2]. They proved that the MCA method for the detection of NDM strains has high efficiency and precision, which is best achieved by adjusting the primer dilutions.

Morever, three NDM-1 primers with different amplicon length and MBL primers were used to detect $P$. aeruginosa PASGNDM699 strain. Based on Melting temperatures of different dilutions of standard DAN, melt curves of gene amplification were equal to $87.7 \pm 0.5^{\circ} \mathrm{C}, 76.6 \pm 0.5^{\circ} \mathrm{C}, 82.6 \pm 0.5^{\circ} \mathrm{C}$ for NDM- 1 primers, and $90.0 \pm 0.5^{\circ} \mathrm{C}$ for b/aVIM, $90.0 \pm 0.5^{\circ} \mathrm{C}$ for blaSIM, $90.0 \pm 0.5^{\circ} \mathrm{C}$ for blaSIM primers for MBL genes, respectively. This indicated that the specificity of primers with $5^{\circ} \mathrm{C}$ error range can detect $P$. aeruginosa PASGNDM699 strains. Andini et al[23] showed that accurate analysis of the melting curve could play a very important role in the diagnosis. Ashrafi et al [10]found that to obtain the best performance in sophisticated methods such as HRM, the melting temperature of DNA must be monitored in various dilutions to obtain accurate sensitivity and specificity. Tahmasebi et al[7] also conformed that efficiency is probably due to the shorter length of products of primers, which enabled better amplification in PCR.

In this study, in Figure 1 showed that for the NDM-1 primer set with 55bp, the linear range was determined to extend as low as $10^{0} \mathrm{CFU} / \mathrm{mL}, N D M-1$ with $85 \mathrm{bp}$ was $10^{3} \mathrm{CFU} / \mathrm{mL}$ and $N D M-1$ with $155 \mathrm{bp}$ was $10^{1}$ $\mathrm{CFU} / \mathrm{mL}$ as indicated by the lowest DNA concentration value on each of the standard curves. Further, Figure 2 indicates that for the blaSHV primer set, the linear range was determined to extend as low as $10^{0}$ $\mathrm{CFU} / \mathrm{mL}$, blaSPM was $10^{3} \mathrm{CFU} / \mathrm{mL}$ and blaVIM was $10^{1} \mathrm{CFU} / \mathrm{mL}$. This is consistent with observation of Smiljanic et al[24]. They illustrated that identification of NDM and MBL strains in Gram-negative nonfermentative is difficult, because the resistance to carbapenems in these bacteria is encoded by similar sequences. Thus, the use of a sensitive and precise method such as HRM along with specific primers could enable the identification of strains such as MBL and NDM.

In the present study, HRM and different primers were used to identify P. aeruginosa PASGNDM699 strain. In a study,by Ding et al.[25] Proposed the resistance of PASGNDM699 strain to a wide range of antibiotics. They also confirmed the clinical importance of PASGNDM strains in causing resistant infections. Based on Figure 4 and 5, all the DNA dilutions of $P$. aeruginosa PASGNDM699 strain were identified (dilution $10^{8}$ to $10^{0} \mathrm{CFU} / \mathrm{mL}$ ). The results were different from the obtained by Naas et al[26] and 
Smiljanic et al[27] studies. They confirmed the identification of NDM strains at dilution 103 and stated that the HRM method had limitations in the detection of different dilutions. Although identification of MBL and NDM strains has been performed in various studies in Sweden[28], USA[29], Australia[30], and Italy[31] in gram-negative Bacteria by HRM method, the novelty of the present study was the use of HRM method to identify P. aeruginosa PASGNDM699 strain. It was also found that the HRM method is highly potent in detecting PASGNDM699 strains that are resistant to colistin and carbapenem.

According to our results, the short primers ( $\mathrm{N}-1$ with 55bp and blaVIm with $111 \mathrm{bp}$ ) had the best sensitivity and specificity in the HRM assay, in addition, they identified the NDM-1 and MBL genes in all dilutions. On the other hand, the purity of the extracted DNA was also a factor. This makes us to think that the amplification of long fragment heavily depends on the DNA quality. Słomka et al[13] demonstrated that when the DNA quality is low, saying DNA degradation or long DNA breaks during extraction makes the long template harder to be amplified. This problem gets worse in digital PCR because the reagent range in the reaction gets more stringent and template is much more diluted. Meanwhile in the digital PCR, as there is only one molecule in the well, the high ratio of primer for short amplicon might not be necessary and even cause the long template can't be amplified because of the primer competition.

However, HRM assay is used to amplify and concurrently quantify a targeted DNA molecule and enables both detection and quantification of DNA. HRM PCR needs a fluorescent reporter that binds to the formed product and reports its presence by fluorescence. The EvaGreen ${ }^{\circledR}$ Dye was used in this study, because it is an saturating dye which do not interfere with PCR reactions, even if they used at the largest level of saturation which gives the maximum fluorescence; That the Eischeid[32] study confirms these results.

It is necessary to point out limitations of the HRM approach in this study. The length of the selected primers should be considered to identify the bacterial sub-strains. If the ratios of the different agents are higher than 1:10, the system does not detect the infectious agent which is in lower quantities. However, the determination of $\mathrm{Tm}$ is very sensitive to the composition of the PCR reaction mixture,especiallyto the ionic strength. To avoid Tm bias due to thepipetting errors between PCR runs, the application of mastermixes is recommended. Limitation of the method can be thatvarious mastermixes offered by differentsuppliers vary in reagent composition. This may influence the Tm values. Besides, in case of different mastermixes from different suppliers, calibration is necessary to establish the newTmdata on the fungal strains.

In conclusion, we demonstrated that the HRM assay is a rapid and sensitive pre-sequence screening tool which allows the detection of point low concetration of a DNA. It eliminates much of the labour and cost involved in performing DNA sequencing of an entire gene and direct DNA sequencing is therefore only required as confirmation of a mutation or polymorphism. Compared with existing methods it is not only more cost-effective, but is also capable of detecting new functional mutations that will have importance in cascade screening of affected subjects. Finally, the analysis of the melting curves is an important step in the identification of heterozygous base changes. The selection of the melting temperature range is 
important to the analysis as there needs to be sufficient data both prior to and following the melting transition, to allow reliable normalisation of the melting curves. HRM has also the capacity to greatly increase the scope and sensitivity of haplotype analysis.

\section{Abbreviations}

HRM: High Resolution Melting Analysis; SIM: Seoul IMipenemase; VIM: Verona integron-borne metallo- $\beta$ lactamase; NDM: Extended-spectrum $\beta$-lactamases; MBL: Metallo- $\beta$-Lactamase; SMP: Sao Paulo metallo$\beta$-lactamase; HRM: High Resolution Melting Analysis; Tm: Temperature; CT: Cycle Threshold.

\section{Declarations}

\section{Ethics approval and consent to participate}

This study was approved by the ethics committee of Hamadan University of Medical Sciences ((No: IR.UMSHA.REC.1396.637).Consent for publication Not applicable

\section{Availability of data and materials}

The data can be accessible to the interested researchers by the corresponding authors on reasonable request.

\section{Consent for publication}

Not Applicable.

\section{Funding}

The Research and technology of Vice Chancellor of Hamadan University of Medical Sciences (Project number: 9609286081).

\section{Competing interests}

The authors declare that they have no competing interests.

\section{Acknowledgments}

The authors would like to acknowledge the Vice Chancellor of Hamadan University of Medical Sciences for the funding and support of the study. Funding This study has been adapted from a reserch fund at Hamadan University of Medical Sciences (Project No: 9609286081).

\section{Authors' contributions}

MRA and HT proposed, designed, carried out the study, HT and SD analyzed the generated data and drafted the manuscript and performed the data analysis. MRA provided with some of the strains and HT 
participated in proofreading of the manuscript and in critical revision. All authors read and approved the fnal manuscript.

\section{Author details}

${ }^{1}$ Microbiology department, Faculty of Medicine, Hamadan University of Medical Sciences, Hamadan, Iran. ${ }^{2}$ Microbiology department, Faculty of Medicine, Zahedan University of Medical Sciences, Zahedan, Iran.

\section{References}

1. Xu Z, Xie J, Soteyome T, Peters BM, Shirtliff ME, Liu J, Harro JM. Polymicrobial interaction and biofilms between Staphylococcus aureus and Pseudomonas aeruginosa: an underestimated concern in food safety. Curr Opin Food Sci. 2019;26:57-64.

2. Mentasti M, Prime K, Sands K, Khan S, Wootton M. Rapid detection of IMP, NDM, VIM, KPC and OXA48-like carbapenemases from Enterobacteriales and Gram-negative non-fermenter bacteria by realtime PCR and melt-curve analysis. Eur J Clin Microbiol Infect Dis. 2019;38:2029-36.

3. Kaur A, Singh S. Prevalence of Extended Spectrum Betalactamase (ESBL) and Metallobetalactamase (MBL) Producing Pseudomonas aeruginosa and Acinetobacter baumannii Isolated from Various Clinical Samples. J Pathog. 2018;2018:7.

4. Khorvash F, Yazdani M, Shabani S, Soudi A. Pseudomonas aeruginosa-producing Metallo- $\beta$ lactamases (VIM, IMP, SME, and AIM) in the Clinical Isolates of Intensive Care Units, a University Hospital in Isfahan, Iran. Advanced Biomedical Research. 2017;6:147.

5. Tahmasebi H, Maleki F, Dehbashi S, Arabestani M. Role and Function of KPC and MBL Enzymes in Increasing the Pathogenicity of Pseudomonas Aeruginosa Isolated from Burn Wounds. J Babol Univ Med Sci. 2019;21:127-34.

6. Bordin A, Trembizki E, Windsor M, Wee R, Tan LY, Buckley C, Syrmis M, Bergh H, Cottrell K, Zowawi HM, Sidjabat HE, Harris PNA, Nimmo GR, Paterson DL, Whiley DM. Evaluation of the SpeeDx Carba (beta) multiplex real-time PCR assay for detection of NDM, KPC, OXA-48-like, IMP-4-like and VIM carbapenemase genes. BMC Infect Dis. 2019;19:571.

7. Tahmasebi H, Dehbashi S, Arabestani MR. High resolution melting curve analysis method for detecting of carbapenemases producing pseudomonas aeruginosa. Journal of Krishna Institute of Medical Sciences University. 2018;7:70-7.

8. Dehbashi S, Tahmasebi H, Arabestani MR. The Clinical Utility of Analysis High Resolution Melting Curve Assay for Simultaneous Identification of Methicillin and Mupirocin Resistant in CoagulaseNegative Staphylococci. Clin Lab. 2019;65.

9. Landolt P, Stephan R, Scherrer S. Development of a new High Resolution Melting (HRM) assay for identification and differentiation of Mycobacterium tuberculosis complex samples. Sci Rep. 2019;9:1850. 
10. Ashrafi R, Bruneaux M, Sundberg L-R, Pulkkinen K, Ketola T. Application of high resolution melting assay (HRM) to study temperature-dependent intraspecific competition in a pathogenic bacterium. Sci Rep. 2017;7:980-

11. Tamburro M, Ripabelli G. High Resolution Melting as a rapid, reliable, accurate and cost-effective emerging tool for genotyping pathogenic bacteria and enhancing molecular epidemiological surveillance: a comprehensive review of the literature. Ann Ig. 2017;29:293-316.

12. Wong YP, Chua KH, Thong KL. One-step species-specific high resolution melting analysis for nosocomial bacteria detection. J Microbiol Methods. 2014;107:133-7.

13. Słomka M, Sobalska-Kwapis M, Wachulec M, Bartosz G, Strapagiel D. High Resolution Melting (HRM) for High-Throughput Genotyping-Limitations and Caveats in Practical Case Studies. Int J Mol Sci. 2017;18:2316.

14. Tahmasebi H, Dehbashi S, Arabestani MR. Identification of gene mutation patterns obtained from resistance to mupirocin in methicillin-resistant staphylococcus aureus clinical strains, using highresolution melting (HRM) method. J Isfahan Med Sch. 2018;36(476):403-10.

15. Bodnar GC, Martins HM, De Oliveira CF, Morey AT, Tavares ER, Cardoso JD, Perugini MR, Lioni LM, Yamada-Ogatta SF, Kobayashi RK, Nakazato G. Comparison of HRM analysis and three REP-PCR genomic fingerprint methods for rapid typing of MRSA at a Brazilian hospital. J Infect Dev Ctries. 2016;10:1306-17

16. Ly TDA, Hadjadj L, Hoang VT, Louni M, Dao TL, Badiaga S, Tissot-Dupont H, Raoult D, Rolain J-M, Gautret P. Low prevalence of resistance genes in sheltered homeless population in Marseille, France, 2014-2018. Infect Drug Resist. 2019;12:1139-51.

17. Monteiro J, Widen RH, Pignatari AC, Kubasek C, Silbert S. Rapid detection of carbapenemase genes by multiplex real-time PCR. J Antimicrob Chemother. 2012;67:906-9.

18. Kosykowska E, Dzieciątkowski T, Mlynarczyk G. Rapid Detection of NDM, VIM, KPC and IMP Carbapenemases by Real-Time PCR. J Bacteriol Parasitol. 2016;07.

19. Alkasaby NM, El Sayed Zaki M. Molecular Study of Acinetobacter baumannii Isolates for Metallo- $\beta$ Lactamases and Extended-Spectrum- $\beta$-Lactamases Genes in Intensive Care Unit, Mansoura University Hospital, Egypt. Int J Microbiol. 2017;2017:6.

20. Pfaffl MW. A new mathematical model for relative quantification in real-time RT-PCR. Nucleic Acids Res. 2001;29:e45.

21. Lalonde LF, Reyes J, Gajadhar AA. Application of a qPCR assay with melting curve analysis for detection and differentiation of protozoan oocysts in human fecal samples from Dominican Republic. Am J Trop Med Hyg. 2013;89:892-8.

22. Heydari N, Alikhani MY, Tahmasebi H, Asghari B, Arabestani MR. Design of Melting Curve Analysis (MCA) by Real-Time Polymerase Chain Reaction Assay for Rapid Distinction of Staphylococci and Antibiotic Resistance. Arch Clin Infect Dis. 2019;14:e81604.

23. Andini N, Wang B, Athamanolap P, Hardick J, Masek BJ, Thair S, Hu A, Avornu G, Peterson S, Cogill S, Rothman RE, Carroll KC, Gaydos CA, Wang JT-H, Batzoglou S, Yang S. Microbial Typing by Machine 
Learned DNA Melt Signatures. Sci Rep. 2017;7:42097.

24. Smiljanic M, Kaase M, Ahmad-Nejad P, Ghebremedhin B. Comparison of in-house and commercial real time-PCR based carbapenemase gene detection methods in Enterobacteriaceae and nonfermenting gram-negative bacterial isolates. Ann Clin Microbiol Antimicrob. 2017;16:48.

25. Ding Y, Teo JWP, Drautz-Moses DI, Schuster SC, Givskov M, Yang L. Acquisition of resistance to carbapenem and macrolide-mediated quorum sensing inhibition by Pseudomonas aeruginosa via ICE(Tn4371) 6385. Communications biology. 2018;1:57.

26. Naas T, Ergani A, Carrër A, Nordmann P. Real-time PCR for detection of NDM-1 carbapenemase genes from spiked stool samples. Antimicrob Agents Chemother. 2011;55:4038-43.

27. Smiljanic M, Kaase M, Ahmad-Nejad P, Ghebremedhin B. Comparison of in-house and commercial real time-PCR based carbapenemase gene detection methods in Enterobacteriaceae and nonfermenting gram-negative bacterial isolates. Ann Clin Microbiol Antimicrob. 2017;16:48-.

28. Woksepp H, Ryberg A, Billström H, Hällgren A, Nilsson LE, Marklund B-I, Olsson-Liljequist B, Schön T. Evaluation of high-resolution melting curve analysis of ligation-mediated real-time PCR, a rapid method for epidemiological typing of ESKAPE (Enterococcus faecium, Staphylococcus aureus, Klebsiella pneumoniae, Acinetobacter baumannii, Pseudomonas aeruginosa, and Enterobacter Species) pathogens. J Clin Microbiol. 2014;52:4339-42.

29. Hemarajata P, Yang S, Hindler JA, Humphries RM. Development of a novel real-time PCR assay with high-resolution melt analysis to detect and differentiate OXA-48-Like $\beta$-lactamases in carbapenemresistant Enterobacteriaceae. Antimicrob Agents Chemother. 2015;59:5574-80.

30. Bordin A, Trembizki E, Windsor M, Wee R, Tan LY, Buckley C, Syrmis M, Bergh H, Cottrell K, Zowawi HM, Sidjabat HE, Harris PNA, Nimmo GR, Paterson DL, Whiley DM. Evaluation of the SpeeDx Carba (beta) multiplex real-time PCR assay for detection of NDM, KPC, OXA-48-like, IMP-4-like and VIM carbapenemase genes. BMC Infect Dis. 2019;19:571.

31. Gori A, Cerboneschi M, Tegli S. High-Resolution Melting Analysis as a Powerful Tool to Discriminate and Genotype Pseudomonas savastanoi Pathovars and Strains. PLOS ONE. 2012;7:e30199.

32. Eischeid AC. SYTO dyes and EvaGreen outperform SYBR Green in real-time PCR. BMC Res Notes. 2011;4:263-.

\section{Table}


Table 1. Oligonucleotide sequences used in this study

\begin{tabular}{|c|c|c|c|c|c|}
\hline Toreget & Primer Name & Sequence of Primers & Melting Tm & Product size $(\mathrm{bp})$ & References \\
\hline & $N-1$ & F: GACCGCCCAGATCCTCAA & 87.57 & 55 & {$[6]$} \\
\hline & & R: CGCGACCGGCAGGTT & & & \\
\hline & $N-2$ & F: TTGGCCTTGCTGTCCTTG & 76.92 & 85 & [17] \\
\hline \multirow[t]{5}{*}{ NDM-1 } & & R: ACACCAGTGACAATATCACCG & & & \\
\hline & $N-3$ & F: GCGCAACACAGCCTGACTTT & 82.97 & 155 & [16] \\
\hline & & R: CAGCCACCAAAAGCGATGTC & & & \\
\hline & blaSIM & TACAAGGGATTCGGCATCG & 85.35 & 577 & [19] \\
\hline & & TAATGGCCTGTTCCCATGTG & & & \\
\hline \multirow[t]{4}{*}{ MBL } & blaVIM & F: TCTCCACGCACTTTCATGAC & 84.56 & 124 & [18] \\
\hline & & R: GTGGGAATCTCGTTCCCCTC & & & \\
\hline & blaSPM & F: AAAATCTGGGTACGCAAACG & 86.62 & 271 & [19] \\
\hline & & R: ACATTATCCGCTGGAACAGG & & & \\
\hline
\end{tabular}

\section{Figures}
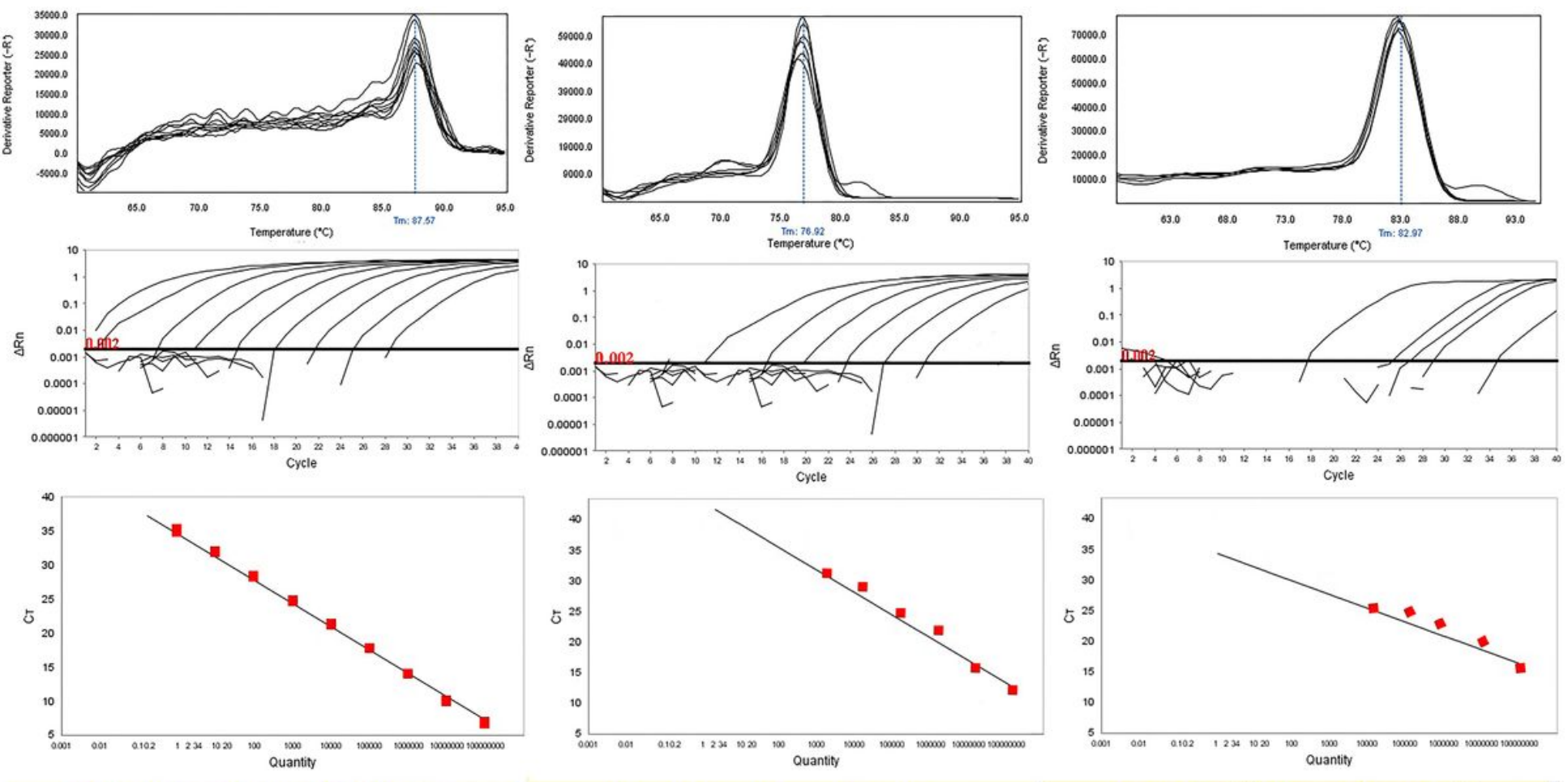

Target:N-1 Slope:-3.111 Y.Inter: $34.638 \underline{R}^{2}: 0.994$ Eff;: 109.623

Target:N-2 Slope:-3.275 Y.Inter; $31.917 R^{2}: 0.976$ Effr; 101.988

Iarget:N3 Slope:-3.351 Y.-Inter:35.192 $R^{2}: 0.958$ Efff; $0.98 .8 \quad \hat{v}$

\section{Figure 1}

Analytical sensitivity of Real-Time PCR and examples of optimization of primer pairs based on melting curve analysis for NDM primers used to detect P. aeruginosa PASGNDM699. The melting curves for each primer pair were investigated. Right: $\mathrm{N}-1$ gene with a melting point of $87.53 \pm 0 / 50 \mathrm{C}$, Middle: $\mathrm{N}-2$ gene with a melting point of $76.92 \pm 0 / 5 \mathrm{oC}$; Left: $\mathrm{N}-3$ gene with a melting point of $82.97 \pm 0 / 5 \mathrm{oC}$. The mean of a: 108 ; 
b: 107; c: 106; d: 105; e: 104; f: 103; g: 102; h: 101 and i: 100 CFU/mL of DNA dilutions. Bold black Horizontal lines represent cycle threshold of Real Time PCR. One peak with a shoulder corresponds to genomic DNA amplification; no peak corresponds to no amplification. Eva Green color and single tube reaction were used in this test. Also, Real Time PCR was performed as single-step.
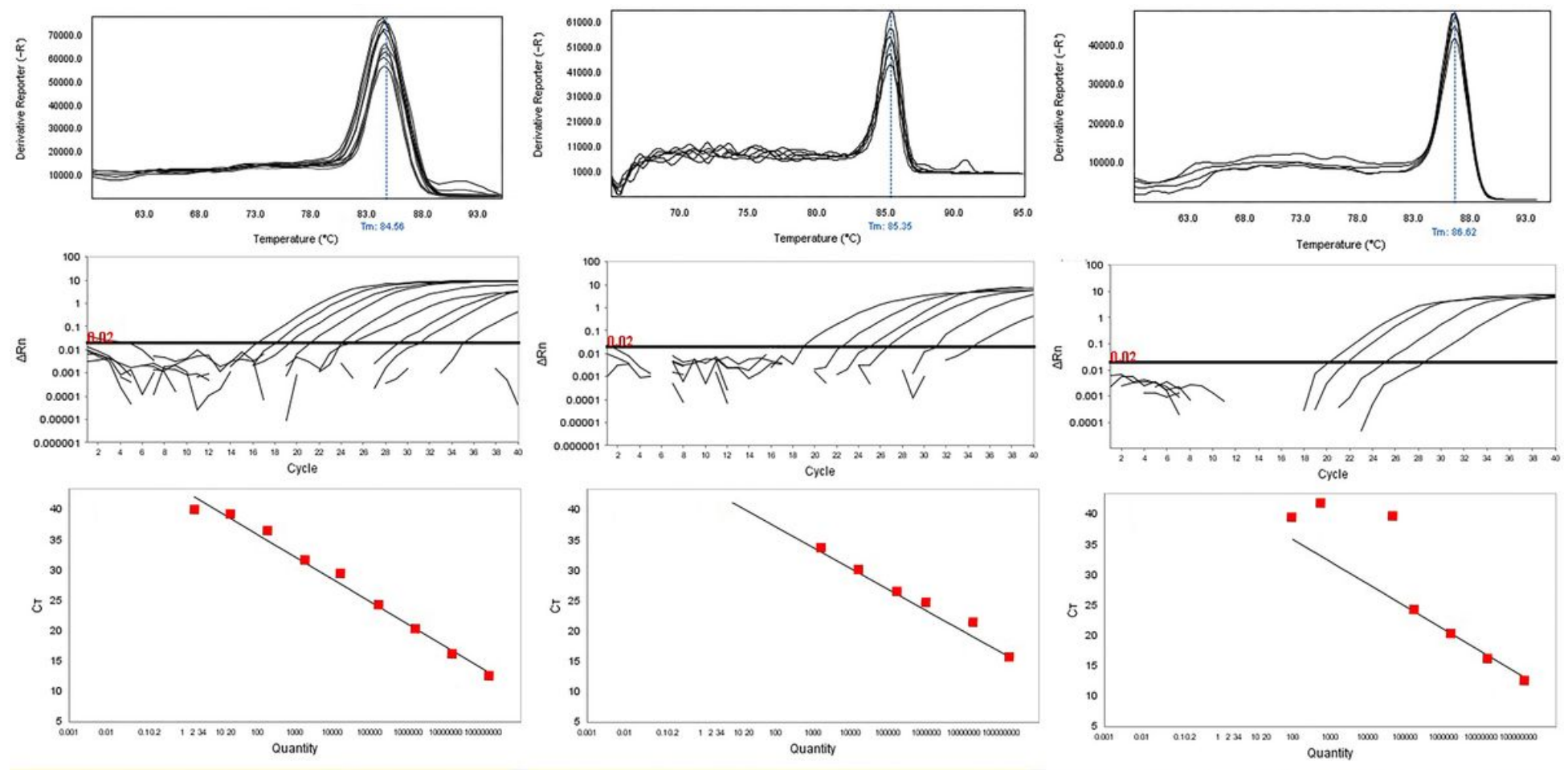

Target:blaVIM Slope:-3.112 Y..Inter:33.125 $\mathrm{R}^{2}: 0.999$ EtfS:109.573

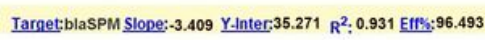

$\hat{\imath}$

\section{Figure 2}

Analytical sensitivity of Real-Time PCR and examples of optimization of primer pairs based on melting curve analysis for MBL primers used to detect P. aeruginosa PASGNDM699. The melting curves for each primer pair were investigated. Left: blaVIM gene with a melting point of $84.56 \pm 0 / 50 \mathrm{C}$, Middle: blaSPM gene with a melting point of $85.35 \pm 0 / 5 \mathrm{oC}$; Right: blaSIM gene with a melting point of $86.62 \pm 0 / 5 \mathrm{oC}$. The mean of a: 108; b: 107; c: 106; d: 105; e: 104; f: 103; g: 102; h: 101 and i: 100 CFU/mL of DNA dilutions. Bold black Horizontal lines represent cycle threshold of Real Time PCR. One peak with a shoulder corresponds to genomic DNA amplification; no peak corresponds to no amplification. Eva Green color and single tube reaction were used in this test. Also, Real Time PCR was performed as single-step. 

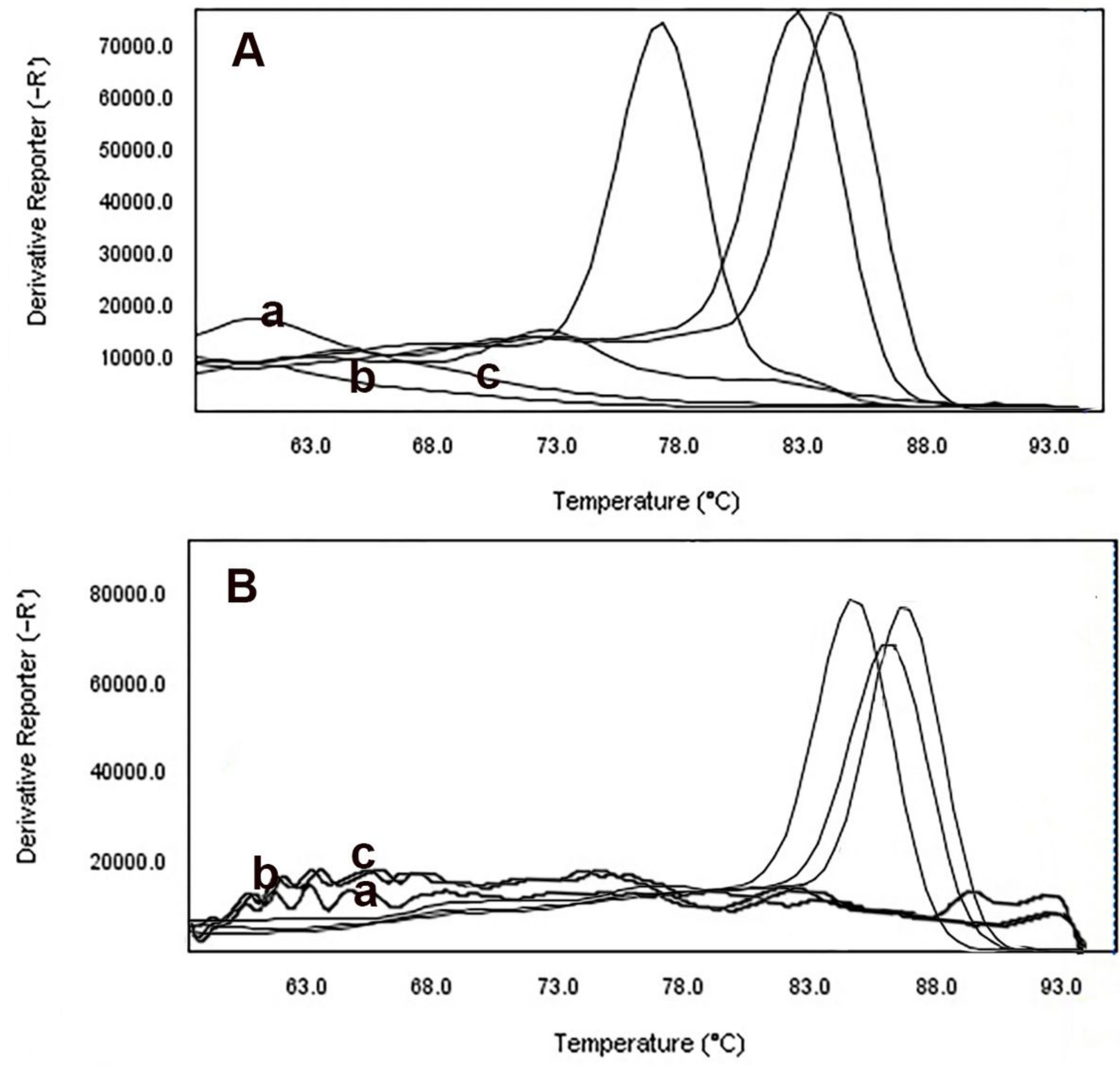

Figure 3

Melting curve analysis and analytical specificity of Real-Time PCR for NDM primers (A) and MBL primers (B) used to detect P. aeruginosa PASGNDM699. a: Blank tube; b: Enterococcus faecalis NCTC13779; and C: Staphylococcus aureus ATCC25923. One peak with a shoulder corresponds to genomic DNA amplification; no peak corresponds to no amplification. Eva Green color and single tube reaction were used in this test. Also, Real Time PCR was performed as single-step. 0.5-McFarland concentration (1.5x $108 \mathrm{CFU} / \mathrm{mL}$ of DNA) was used to determine primer specificity. 

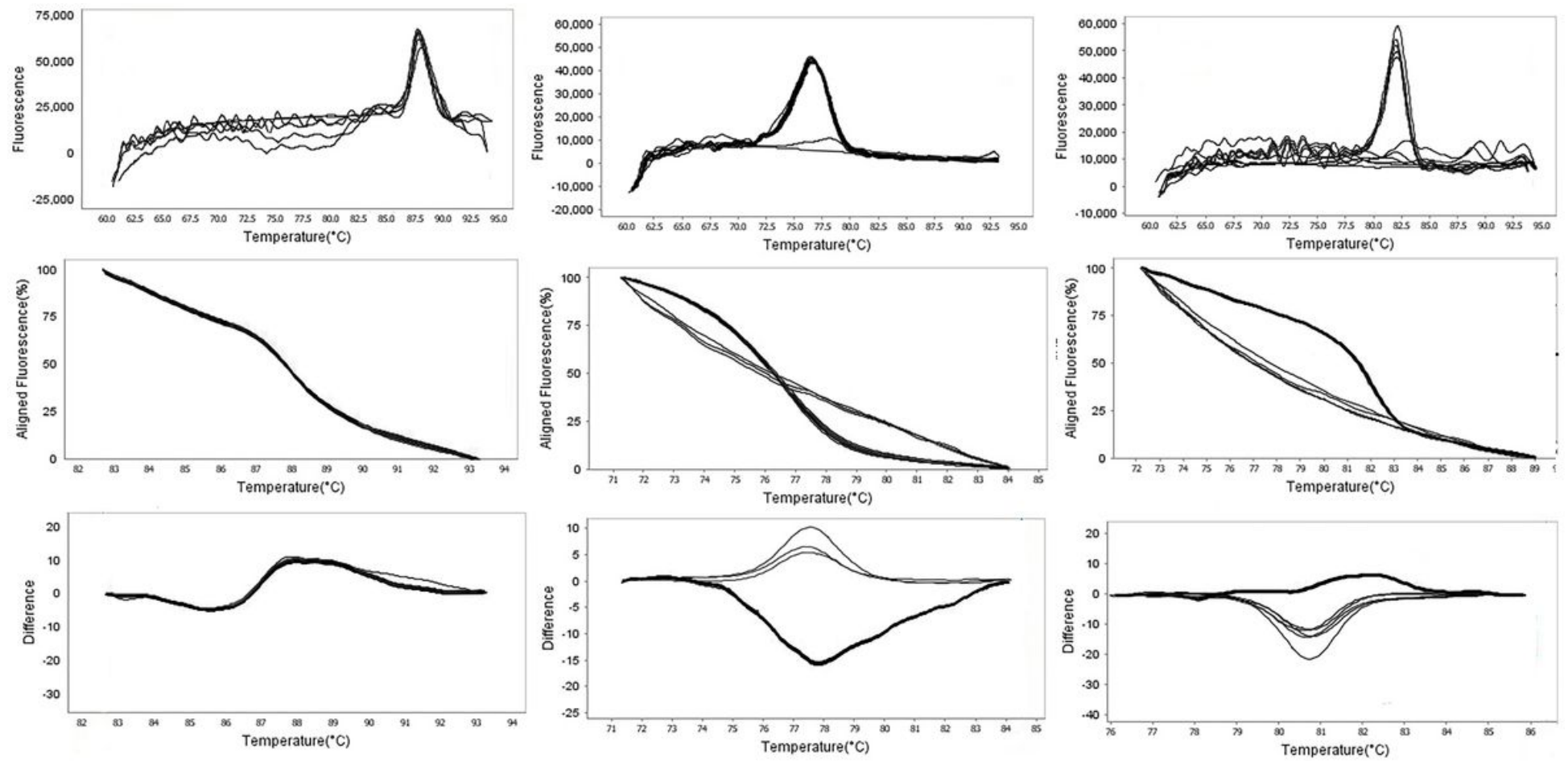

Figure 4

HRM graphs corresponding to one high resolution melting analysis of a subset of P. aeruginosa PASGNDM699 by N-1 (Left), N-2 (Middle) and N-3 (right) genes. DNA samples from all the dilutions involved in this study were prepared and amplified successfully using the EvaGreen dye-based method in the $A B I$ instrument. Primers specific melting peaks $(T m)$ were obtained via HRM analysis, allowing the differentiation of all investigated $\beta$-lactamase enzymes. Due to the highly saturating EvaGreen dye and the HRM analysis, the accuracy of the resolution was $\pm 0.1-0.5^{\circ} \mathrm{C}$.
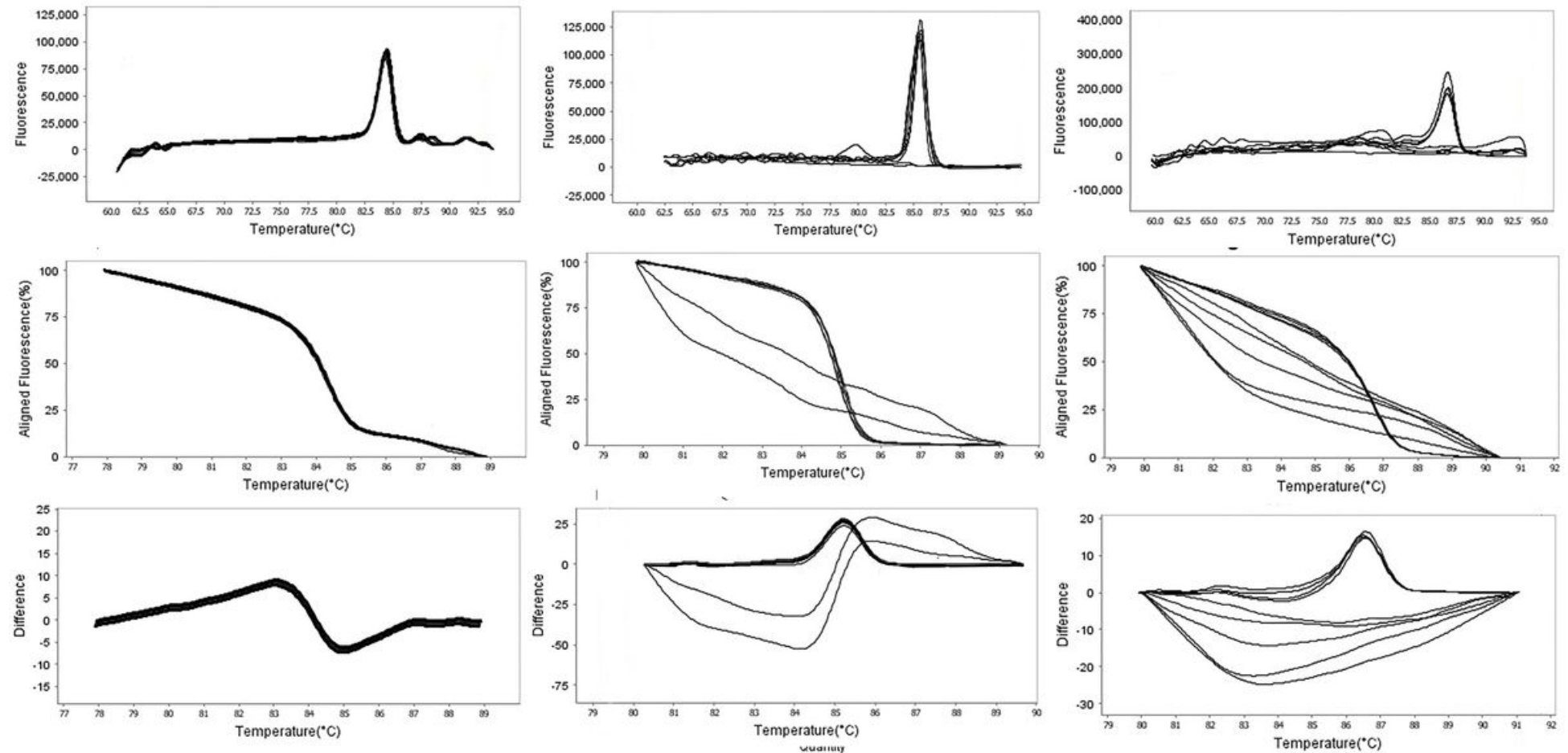


\section{Figure 5}

HRM graphs corresponding to one high resolution melting analysis of a subset of P. aeruginosa PASGNDM699 by blaVIM (Left), blaSIM (Middle) and blaSPM (right) genes. DNA samples from all the dilutions involved in this study were prepared and amplified successfully using the EvaGreen dye-based method in the $A B I$ instrument. Primers specific melting peaks $(\mathrm{Tm})$ were obtained via HRM analysis, allowing the differentiation of all investigated $\beta$-lactamase enzymes. Due to the highly saturating EvaGreen dye and the HRM analysis, the accuracy of the resolution was $\pm 0.1^{\circ} \mathrm{C}-0.5^{\circ} \mathrm{C}$. 